\title{
Solar neutrino experiments and Borexino perspectives
}

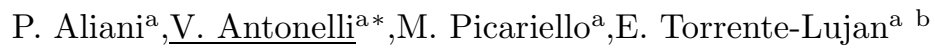 \\ ${ }^{\text {a} D i p . ~ d i ~ F i s i c a, ~ U n i v e r s i t a ̀ ~ d i ~ M i l a n o ~ e ~ I . N . F . N ., ~ S e z i o n e ~ d i ~ M i l a n o, ~ V i a ~ C e l o r i a ~ 16, ~ M i l a n o, ~ I t a l y ~}$ \\ ${ }^{\text {b}}$ Dept. Fisica Teorica,C-XI, Univ. Aut. de Madrid, Spain and CERN TH-Division, CH-1202 Geneve
}

We present an updated analysis of all the data available about solar neutrinos, including the charged current SNO results. The best fit of the data is obtained in the Large Mixing Angle region, but different solutions are still possible. We also study the perspectives of Borexino and conclude that this experiment, with a parallel analysis of total rate and day-night asymmetry, should be able to discriminate between the different possible solutions.

\section{INTRODUCTION}

Fifty years after neutrino discovery, many of its properties (like its mass) are not completly understood [1]. Many experiments tried to answer these questions using different techniques. Here we focus our attention on solar neutrinos. The SNO experiment [2] measured the ${ }^{8} \mathrm{~B}$ Solar neutrinos through the two reactions: (1) charged current (CC): $\nu_{e}+d \rightarrow 2 p+e^{-}$and (2) elastic scattering (ES): $\nu_{x}+e^{-} \rightarrow \nu_{x}+e^{-}$. The results [2] confirmed the previous evidences [3] that the flux of $\nu_{e}$ reaching the Earth is less than the Solar Standard Model (SSM) prediction [4,5]. Comparing the two channels, SNO also gave a strong confirmation of the validity of $\nu$ oscillation hypothesis and it can be considered the first demonstration of the appearance of muon and tau neutrinos detected at the Earth. In this work we restrict the analysis to the bidimensional case for simplicity,but the extensions to more than two flavours will be treated elsewhere [6]. The interest in neutrino physics is justfied not only by the data available, but also by the well founded hope that the forthcoming experiments (like Borexino [7] and the long baseline experiments) will be able to discriminate more clearly between the possible solutions of the solar neutrino problem [8]. The first aim of our work is to produce a phenomenological analysis of all the available solar neutrino data. We determine the values of the mixing parameters compatible with the data and compare the allowed regions with the ones selected from

\footnotetext{
*talk given by V. Antonelli
}

Borexino, depending on the signal it will find. The analysis can be divided in the following steps. We first compute exactly, using an evolution operator formalism [9], the survival probability that a neutrino produced with a well determined flavour is still of the same kind or has changed flavour when it arrives at the detector. To take into account the interaction with the Earth, we assume a spherical model 10 in which the Earth is divided in eleven radial density zones. The other building block of the analysis is the study of the different aspects of each experiment, as the cross section for the interaction of the neutrino in the detector 11], the detector resolution and its efficiency. More information about this and other points of analysis are reported in [12]. We obtain a response function for every experiment. From the convolution of survival probability, response function and $\nu$ flux we obtain the expected signal for every experiment and the ratio between this value and the one predicted by the SSM in absence of oscillations.

\section{STATISTICAL ANALYSIS AND RE- SULTS}

For an exhaustive description of the statistical analysis and of our results we refer the interested reader to [12]. Here we just report the salient points. In the most simple case, one includes in the $\chi^{2}$ analysis only the values of the global rates for all the experiments. The global $\chi^{2}$ function is simply defined as:

$\chi_{\mathrm{gl}}^{2}=\left(\boldsymbol{R}^{\text {th }}-\boldsymbol{R}^{\mathrm{exp}}\right)^{T}\left(\sigma^{2}\right)^{-1}\left(\boldsymbol{R}^{\mathrm{th}}-\boldsymbol{R}^{\exp }\right)$

where the covariance matrix $\sigma$ is made up by 
a diagonal part (theoretical, statistical and uncorrelated errors) and another part (correlated systematic uncertainties). The $\boldsymbol{R}^{\text {th,exp }}$ vectors contain the data normalized to the SSM expectations: $R_{i}^{t h, \exp }=S_{i}^{t h, \exp } / S_{i}^{S S M}$, where the index $i$ denotes the different Solar experiments: Chlorine $(\mathrm{Cl})$, Gallium $(\mathrm{Ga})$, SuperKamiokande (SK) and charged current SNO (CC-SNO). The correlation matrices, both including and excluding SNO, are computed using standard techniques [13]. We perform a minimization of the $\chi_{g l}^{2}$ as a function of the oscillation parameters. A point in parameter space $\left(\Delta m^{2}, \tan ^{2} \theta\right)$ is allowed if the globally subtracted $\chi_{g l}^{2}$ fulfills the condition: $\chi_{g l}^{2}\left(\Delta m^{2}, \theta\right)-\chi_{\min }^{2}<\chi_{n}^{2}(C L)$. Where $\chi_{n=2}^{2}$ are the $n=2$ degrees of freedom quantiles.
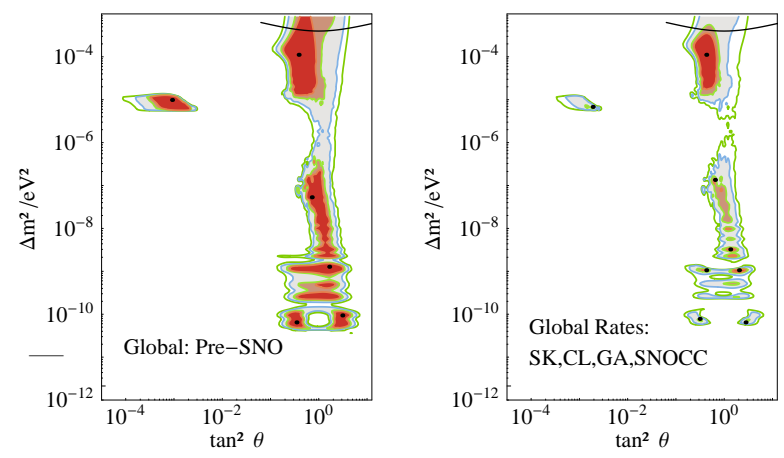

Figure 1. (Left) Global rate analysis from SK,Cl and Ga experiments. (Right) Same analysis including CC-SNO. The black dots are the best fit points; the coloured areas are the allowed regions at 90,95,99 and $99.7 \%$ CL. The region above the solid line is excluded by CHOOZ results at $99 \%$ CL 14.

The results of this analysis are represented in Fig. 1. One can distinguish 4 different regions: Small Mixing Angle (SMA), Large Mixing Angle (LMA), Low mass (LOW) and Vacuum region (VAC). After the introduction of SNO data the different regions become well separated. The best-fit point is no longer the SMA solution, but the one in the LMA region and the statistical significance of the SMA region is drastically reduced. When introducing also the data of the SK energy spectrum rates the statistical analysis becomes more complex. In this case we have 41 experimental data inputs: the $2 \times 19$ values of the bins in which the day and night spectrums are divided, plus the total rates for $\mathrm{Cl}, \mathrm{Ga}$ and CC-SNO. The procedure we adopt to define the $\chi^{2}$ parameter and perform the minimization (see [12]) follows the one used by the SK collaboration. In Table 1 we report the results that one gets in the case in which the ${ }^{8} \mathrm{~B}$ flux is constrained to vary around the BPB2001 [5] central value with the standard deviation given by SSM. The corresponding contour plots are drawn in Fig. 2 together with the Borexino contour lines. The other possibility (free ${ }^{8} \mathrm{~B}$ flux) is discussed in 12].

\section{BOREXINO PERSPECTIVES}
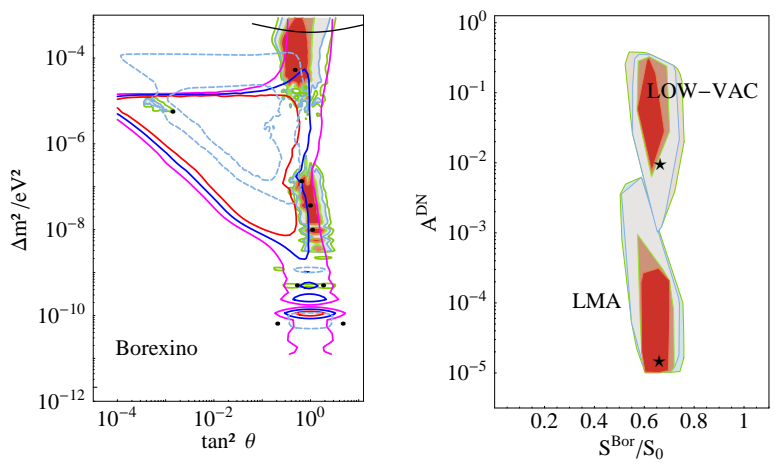

Figure 2. (Left) Contour lines (full lines) for $S^{\text {Bor }} / S_{0}=0.5,0.6,0.7$ superimposed to the contour plots obtained from all other experiments and to the regions from $\mathrm{Cl}$ experiment alone (inside the dashed lines).(Right) Borexino day night asymmetry $\left(A^{D N}\right)$ versus normalized signal $\left(S^{\text {Bor }} / S_{0}\right)$.

We analize the results from all other experiments together with the expectations for Borexino day-night averaged signal normalized with respects to SSM $\left(S^{\text {Bor }} / S_{0}=S^{D-N} / S_{S S M}\right)$ and day-night asymmetry $\left(A^{D N}=2(D-N) /(D+\right.$ $N))$. In Fig. 2 the contour lines corresponding to different possible values of $S^{B o r} / S_{0}$ are superimposed on the allowed regions obtained from the global analysis of the full set of data for the other experiments. The signal discrimination power of the experiment [15] should be sufficient to distinguish between the different allowed regions in the $\left(\Delta m^{2}, \tan ^{2} \theta\right)$ plane or at least to strongly favour one of them. Borexino potentiality becomes even more evident when we look at the day night asymmetry. From the second graph of Fig. 2 we see that the LMA and the LOW regions correspond to quite different values of $A^{D N}$. 
Table 1

Best fit oscillation parameters. The analysis includes the global rates for Cl,Ga and CC-SNO, and the SK day and night energy spectra. The flux normalization is constrained to vary with SSM standard error and the number of d.o.f. is $n=41-4$. Also reported are the values of $\chi^{2}$ minimum per degree of freedom $\left(\chi_{\min }^{2} / n\right)$ and the statistical significance (goodness of fit g.o.f.)

\begin{tabular}{lllll}
\hline Sol & $\Delta m^{2}$ & $\tan ^{2}(\theta)$ & $\chi_{\min }^{2} / n$ & g.o.f \\
\hline LMA & $5.2 \times 10^{-05}$ & 0.47 & 0.8 & 77 \\
LOW & $9.9 \times 10^{-09}$ & 1.03 & 0.9 & 65 \\
LOW & $3.6 \times 10^{-08}$ & 0.97 & 0.9 & 60 \\
VAC & $5.0 \times 10^{-10}$ & 1.86 & 1.1 & 28 \\
VAC & $5.0 \times 10^{-10}$ & 0.52 & 1.1 & 24 \\
SMA & $5.6 \times 10^{-06}$ & $1.32 \times 10^{-3}$ & 1.4 & 3.2 \\
\hline
\end{tabular}

\section{CONCLUSIONS}

We analyzed all the Solar neutrino data available and for the best solutions which fit the present data, we studied Borexino expectations. In the most comprehensive case, global rates plus spectrum, the best fit was obtained in the LMA region. Solutions in the LOW and VAC regions are still possible although much less favoured. The best possible solution in the SMA region gets a low statistical significance. From the study of the expected Borexino normalized signal and day night asymmetry we conclude the following. In the near future, after 2-3 years of data taking, the combined Borexino measurements of the total event rate with an error below $\pm 5-10 \%$ and day-night total rate asymmetry with a precision comparable to that of SK should allow us to discriminate between the Solar neutrino solutions suggested by present data.

\section{ACKNOWLEDGMENTS}

We are really glad to thank R. Ferrari for his continuous scientific and organizative support, that has been essential for this work. We thank the Milano Borexino group, M. Pallavicini and the organizers of TAUP meeting. We acknowledge the financial support of MIUR and CYCIT.

\section{REFERENCES}

1. E. Torrente-Lujan, arXiv:hep-ph/9902339

2. Q. R. Ahmad et al. [SNO Collaboration], Phys. Rev. Lett. 87 (2001) 071301

3. B.T. Cleveland et al., [HOMESTAKE Coll.] Astrophys. J. 496 (98) 505-526; Y. Fukuda et al. [SK. Coll.] Phys. Rev. Lett. 82 (99) 1810 and 2430; J.N. Abdurashitov et al. [SAGE Coll.], Phys. Rev. Lett. 83(23) (99) 4686; M. Altmann et al. [GNO Coll.] Phys. Lett. B 490 (2000) 16-26; W. Hampel et al., [GALLEX Coll.] Phys. Lett. B 447 (99) 127

4. J.N. Bahcall and M.H. Pinsonneault, Rev. Mod. Phys. 67 (95) 781; S. Turck-Chieze, Nucl. Phys. Proc. Suppl. 91 (2001) 73; E. G. Adelberger et al., Rev. Mod. Phys. 70 (98) 1265 ;

5. J. N. Bahcall, M. H. Pinsonneault and S. Basu, Astrophys. J. 555 (2001) 990

6. work in progress

7. E. Meroni, Nucl. Phys. Proc. Suppl. 100 (2001) 42

8. A.Strumia, F.Vissani, JHEP 0111:048 (2001)

9. E. Torrente-Lujan Phys. Rev. D 59 (99) 093006, 073001; Phys. Lett. B 441(98) 305; V. B. Semikoz, E. Torrente-Lujan Nucl. Phys. B 556 (99) 353; E. Torrente-Lujan Phys. Lett. B 494 (2000) 255.

10. I. Mocioiu and R. Shrock, Phys. Rev. D 62 (2000) 053017

11. J. Bahcall, M. Kamionkowsky, A. Sirlin, Phys. Rev. D 51 (1995) 6146; S. Nakamura et al., Phys. Rev. C 63 (2001) 034617

12. P. Aliani, V. Antonelli, M. Picariello, E. Torrente-Lujan arXiv:hep-ph/0111418

13. G.Fogli and E. Lisi, Astrop. Phys. 3(1995)185

14. M. Apollonio et al. [CHOOZ coll.], Phys. Lett. B 466 (99) 415; F. Boehm et al.,Phys. Rev. D62 (2000) 072002

15. M. Giammarchi [Bor. Col.], private comm. 